\title{
A multidisciplinary approach to the management of spinal metastasis: A review article
}

\author{
Siddharth Badve ${ }^{1}$, Arjun Dhawale ${ }^{2}$, Kshitij Chaudhary', Chetan Anchan ${ }^{4}$
}

\section{Abstract}

Spinal metastasis is a frequent occurrence in patients presenting with advanced malignancy. The burden of this condition is on rise, especially with the availability of aggressive treatment regimens for the primary disease and the improvement in the patient survival. Thoracic spine is the most affected region. The likely source of the primary is from the breast, prostate, lung, thyroid, or kidney. Certain hematological and other malignant conditions can also develop an early spinal involvement that requires timely evaluation and management. The goals for the management of the spinal lesion include preservation of the neurological function, pain control, and maintenance of spinal stability. On the whole, the aim of the treatment continues to palliation in majority of the scenarios. The management strategy is based on the factors that include the patient condition, life expectancy, nature of the tumor pathology, extent of spinal cord compression, severity of neurological deficit, pain control, and the effect on spinal stability. A multidisciplinary approach involving medical oncology, radiation oncology, spine surgery, palliative care and other subspecialtiess forms the cornerstone of the management. Although giant strides have been reported in the advancement of the treatment for spinal metastasis, majority of these avenues are beyond the reach of the patient population from the developing societies. Lack of referral facilities, resource constraints, and geographic disparities are major impediments. The lack of awareness and consensus on the management protocols within the treatment team and the medical community in general poses another challenge in providing an acceptable standard of care. This article offers an insight into the principles that guide the management of spinal metastasis. The application of these principles in the background of the resource constraints that are unique to the South Asian population has also been addressed. This is a synopsis on the multidisciplinary approach to the diagnosis and management of spinal metastasis along with the review of the relevant literature.

Keywords: Vertebral metastasis, spinal stability, pathological fracture, spinal cord compression.

\section{Introduction}

Symptomatic spinal metastasis occurs in $20 \%$ of patients with cancer $[1,2]$. The skeletal system is the third most common system to be affected with metastatic disease after the lungs and liver. Spinal metastasis usually occurs from primaries in the breast, prostate, lung, thyroid, and kidney. The thoracic spine is most commonly affected, followed by the lumbosacral spine and cervical spine.
Treatment is almost always palliative and depends on various factors including primary tumor pathology, spinal cord compression, neurological deficit, pain, and spinal stability. Although there is literature on the approach to spinal metastasis, most of the cited studies are performed in developed countries with a robust health infrastructure and the protocols with respect to quality-of-life indices and survival rates are derived and extrapolated from these studies and reviews. Whether these protocols are universally applicable in the large socioeconomically underprivileged, often un-uninsured population in South Asia is debatable. Although there is often help available from government, charitable organizations and hospitals, this may be time dependent. There are few dedicated national and regional cancer centers with facilities to provide

${ }^{1}$ Orthopaedic Spine Surgeon, Musculoskeletal Institute, Geisinger Health System; MS (Orthopedics), Lewistown, Pennsylvania, United States of America,

2Orthopaedic Spine Surgeon, Department of Orthopedics, Sir H.N. Reliance Foundation Hospital, Mumbai, Maharashtra, India,

3Orthopaedic Spine Surgeon, Department of Orthopaedics, P.D. Hinduja National Hospital and Research Centre, Mumbai, Maharashtra, India,

4Orthopaedic Onco-surgeon, Department of Orthopaedics, Sir H.N. Reliance Foundation Hospital, Girgaon, Mumbai, Maharashtra, India

Address of Correspondence

Dr. Siddharth Badve,

Orthopaedic Spine Surgeon, Musculoskeletal Institute, Geisinger Health System; MS (Orthopedics), Lewistown, Pennsylvania, United States of America.

E-mail: siddharthbadve@hotmail.com

Submitted Date: 30-Apr-21, Review Date: 21-May-21, Accepted Date: 28-May-21 \& Published Date: 31-Dec-21

Journal of Clinical Orthopaedics | Available on www.jcorth.com | DOI:10.13107/jcorth.2021.v06i02.444 This is an Open Access article distributed under the terms of the Creative Commons Attribution Non-

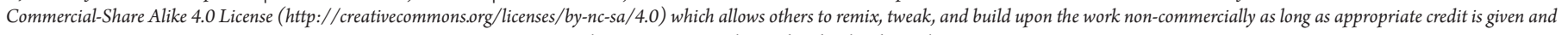
the new creation are licensed under the identical terms. 

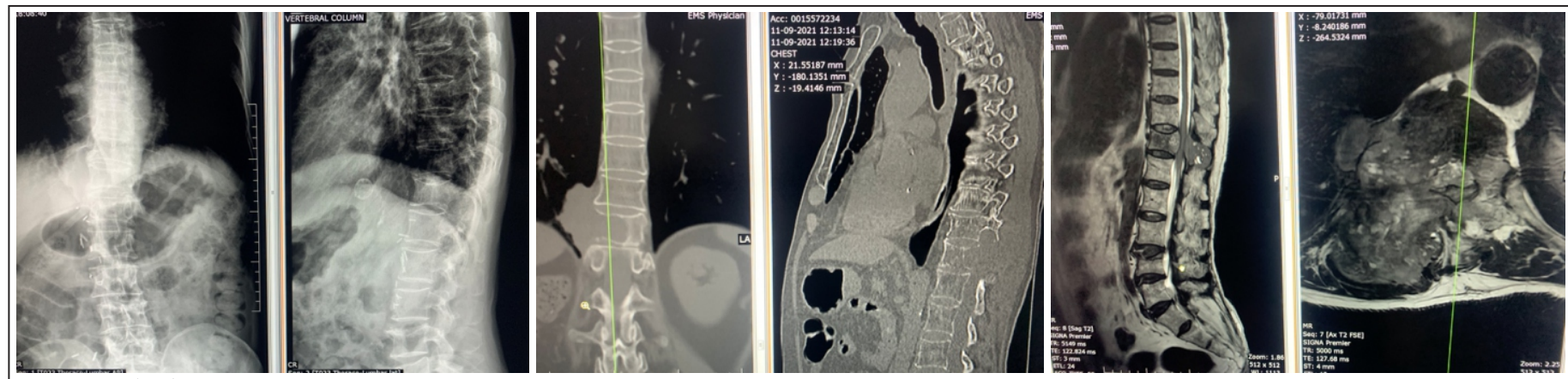

Figure 1: (a-c) 72-year-old male with advanced renal cell carcinoma with right lower limb power grade 5 and left lower limb power grade 0 and preserved sensations with bladder involvement with spinal metastasis involving $\mathrm{L} 1$ with a pathological fracture and epidural mass with spinal cord compression. (a) Radiographs, (b) CT and (C) MRI.

quality treatment at a subsidized cost, but these are often overloaded. In this context, how aggressive palliative treatment should be remains a moot point, and we review the evidence available on the treatment options and protocols for spinal metastasis.

Discussion on the presentation and management of patients with spinal metastasis with review of the pertinent literature

\section{Clinical Presentation}

Pain

Axial pain is usually a presenting characteristic of metastatic spinal disease. Pain in spinal metastasis is often difficult to characterize although it is broadly of two types: mechanical and biologic. The differentiation of the characteristics is important as the character of pain may help to determine treatment choice. Biologic pain is present at rest and usually responds to radiotherapy and steroids. Some patients may present with mechanical pain due to

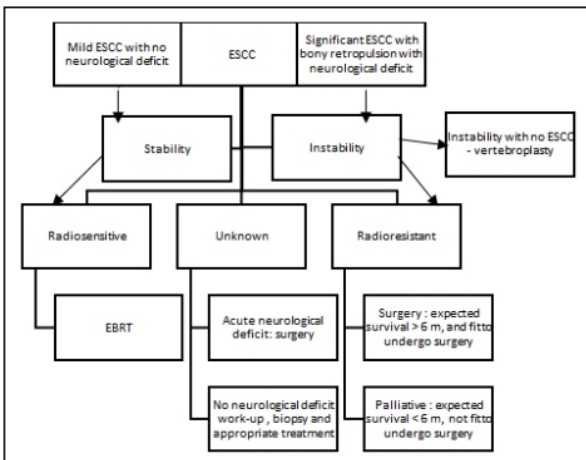

Figure 2: Algorithm for the management of spinal metastasis involving a multidisciplinary approach. pathological fracture which is activity related and subsides with recumbency. These patients may need rest, bracing, or fixation for stabilization.

\section{Neurological symptoms}

Patients may present with focal radiculopathy due to nerve root compression or myelopathy with neurological deficit due to spinal cord compression. Rarely patients may present with bowel, bladder involvement with cauda equina or conus syndrome depending on the levels involved. Neurological symptoms are due to epidural extension of the tumor or pathological fracture of the vertebral body.

\section{Systemic symptoms}

Weight loss, fever, and loss of appetite may be seen although spinal metastasis may remain quiescent and be diagnosed after screening.

\section{Examination}

A thorough systemic examination evaluating possible primary sites such as the thyroid, breast, and lungs with examination of the extremities and joints for appendicular involvement should be performed. The local examination of the entire spine for deformity, local tenderness, and range of movements with a detailed neurological examination including tone, power, reflexes, and sensations will help to identify the level of pathology and differentiate upper motor neuron signs (sensory level of hyperesthesia/hypoesthesia, hyperreflexia, hypertonia, ataxia, and weakness) from the lower motor neuron signs (sensory hypoesthesia, areflexia, and weakness). In early presenters, the neurology may be normal or there may be subtle neurological impairment. Rarely patients may present with dense neurological deficit. Quantifying the neurological involvement with the ASIA or Frankel grades is important. Performance evaluation with scores such as the Karnofsky or Eastern Cooperative Oncology Group to determine the functional disability is important when determining the course of treatment. For example, a moribund patient with poor function and oncology scores would not be suitable for aggressive oncological treatment and would benefit better with palliative pain relief measures.

\section{Workup and Investigations}

The diagnostic work-up of patients with a spinal metastatic lesion includes a series of imaging and blood investigations. The radiological work up can broadly be classified on the basis of whether the lesion is arising from a known malignancy versus a metastatic lesion of unknown origin.

For a cancer patient with a newly developed metastasis, a detailed evaluation to repeat the oncologic staging is mandatory. The usual diagnostic modalities that can be considered include CT scans of the chest, abdomen and pelvis, a bone scan, or alternatively a PET scan. The staging 


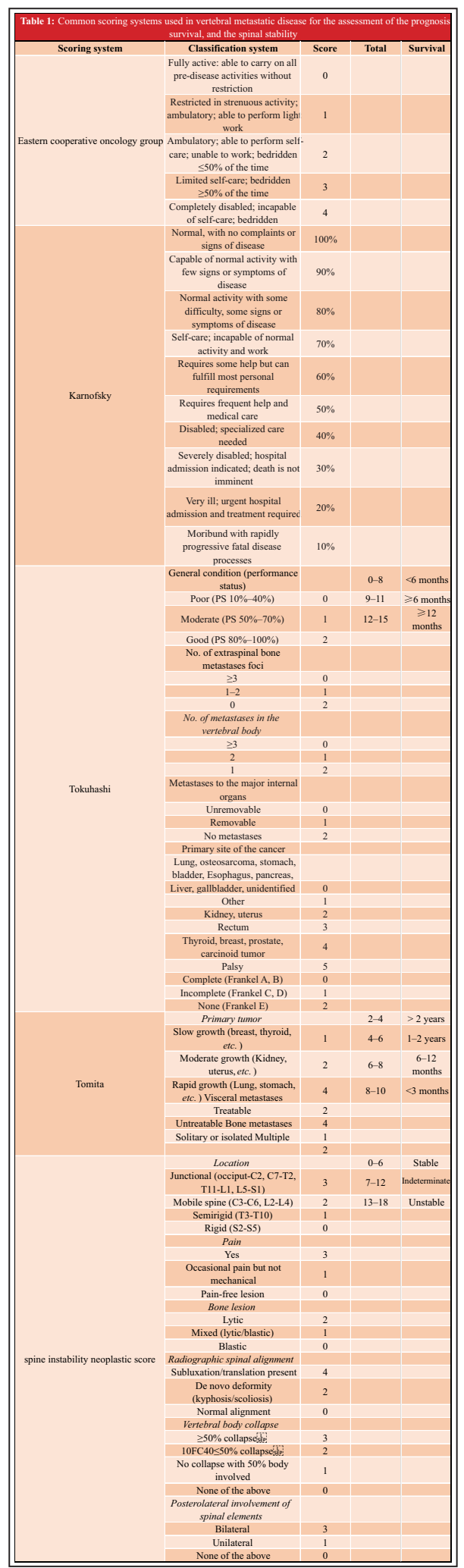

studies may uncover metastatic lesion that may be more easily accessible for the tissue biopsy, in comparison to a spinal lesion. On the contrary, the cancer patients with established metastatic disease, with typical lesion on the radiologic work up may not need a repeat tissue diagnosis.

The typical radiologic work-up for patients with spinal metastasis includes cross-sectional imaging such as MRI, CT scan, and plain radiographs of the spine along with a CT guided biopsy of the lesion. In addition, a CT scan of the chest, abdomen and the pelvis, and a bone scan is helpful to assess the systemic spread. Alternatively, a PET CT offers a similar information concerning the systemic involvement.

\section{Magnetic resonance imaging (MRI) scan}

MRI evaluation of the entire spine is recommended in patients with metastatic spine disease. MRI provides details of metastatic lesion along with the epidural spread of the disease. A combination of sagittal, axial, and coronal sections with $\mathrm{T} 1-$ and $\mathrm{T} 2$ weighted fat saturated or short-tau inversion recovery sequences provide a wealth of information. MRI provides the greatest sensitivity and specificity in the detection of spinal metastases (98.5\% and $98.9 \%$, respectively), with an overall accuracy of 98.7\% [3]. Gadolinium (Gd 153 ) enhancement is useful to evaluate spinal soft tissue, the epidural space, and spinal canal [1]. MRI can be useful in differentiating between osteoporotic compressions fractures resulting from osteoporosis and collapsed metastatic vertebral lesions. Metastatic vertebral lesions may have an appearance of an expanded posterior vertebral cortex, pedicle and posterior element destruction, epidural, paraspinal mass, etc. In contrast to the osteoporotic fracture, the metastatic disease has a tendency to demonstrate diminished signal intensity on $\mathrm{T} 1$ sequences. In addition, on the $\mathrm{T} 2$ sequences, the metastatic lesions are more likely to appear hyperintense in comparison to the bone marrow, with the osteoporotic type being hypointense [2]. The severity of the dural compression and the status of the spinal cord and the neural elements can also be optimally delineated.

\section{Computed tomographic (CT) scan}

The osseous anatomy of the vertebrae is adequately mapped by the use of CT imaging. Addition of myelography can demonstrate the canal compromise and the neurological compression [1]. The delineation of the vertebral anatomy provides an insight in the maintenance of structural integrity and stability of the spinal column. It is a helpful modality in patients with contraindication for MRI [2]. In the evaluation of spinal metastasis, the sensitivity is only $66 \%$ while the diagnostic accuracy is around $89 \%$ [3].

\section{Plain radiography}

It is one of the most commonly used modalities in the evaluation of spinal metastasis, possibly due to ease of availability. It has a low sensitivity as more than $50 \%$ of trabecular bone must be lost for the lesion to be identified or an osteolytic process is evident [4]. The destruction of the unilateral pedicle secondary to the metastatic disease can be evident as the classic "winking owl sign” $[1,2]$. In particular, plan radiographs provide vital information about the overall alignment of the spine. Figure 1 sows 72 -year-old male with advanced renal cell carcinoma with right lower limb power grade 5 and left lower limb power grade 0 and preserved sensations with bladder involvement with spinal metastasis involving L 1 with a pathological fracture and epidural mass with spinal cord compression. (a) Radiographs, (b) CT and (C MRI.

\section{Bone scan}

The radionuclide bone scan using Technetium Tc $99 \mathrm{~m}$ is a well-established modality in the assessment of metastatic skeletal lesions. Except for certain lesions that are purely lytic like multiple myeloma, bone scan has a very high sensitivity in detecting spinal, and other bone metastasis [2]. Moreover, the high sensitivity offers the advantage of 
identifying the lesions up to 18 months in advance in comparison to plain radiography [5]. On the contrary, the low specificity of this investigation can potentially make differentiation with degenerative changes, osteoporotic vertebral insufficiency fracture, and certain inflammatory and infective conditions difficult [2]. It is routinely utilized in evaluating the stage of patients with breast, prostate, lung, kidney, or thyroid malignancies. The protocol of using routine laboratory studies, technetium Tc-99m phosphonate bone scintigraphy, and plain radiography as well as CT of the chest, abdomen, and pelvis can help in identifying the primary site of disease in $85 \%$ of patients who presented with skeletal metastatic disease of unknown origin [6].

\section{Positron emission tomography (PET) - computed tomography scan}

The availability of 5-Flourodeoxyglucose (FDG) PET in combination with the CT in a single scanner has added a unique tool to the evaluation of the patients with metastatic spinal lesions. PET CT can accurately identify and localize metabolically active lesions, both primary and metastatic, thus guiding with the most suitable biopsy site. The tumor cells tend to accumulate FDG unlike the traumatic fractures $[7,8]$. In addition, the assessment of biological response to the treatment as well as early identification and staging of the recurrent lesions is a major advantage. It has been found to be more sensitive in comparison to bone scan and diagnostic $\mathrm{CT}$ in the evaluation of osseous and spinal metastasis. The avidity of even the early metastatic lesions, both the osteoblastic and osteolytic variants to FDG, especially in untreated patients, gives it an advantage over the conventional diagnostic modalities. The sensitivity and the specificity of the PET CT for the detection of an osseous metastasis have been found to be satisfactorily high $[7,8]$.

\section{Image guided biopsy}

CT-guided biopsy is the most crucial investigation in the diagnosis of a spinal metastatic lesion, especially with the background of an unknown primary. It should follow MRI and other radiologic investigation as it can potentially alter the radiologic picture. With the prevalent CT based technology and a transpedicular approach, it is safe and accurate procedure in evaluating spinal lesions in patients with underlying malignancy. The diagnostic accuracy of CT-guided spinal biopsy ranges from $93 \%$ for lytic lesions to $76 \%$ for sclerotic lesions [9]. The diagnostic yield may be improved with the use of large bore needle for the tissue collection. Antibiotics should be withheld before the biopsy so that growth of the potential infective organism is not suppressed. In addition to the histopathological analysis, the sample should be tested for microbial cultures and other investigations considered relevant. An immediate frozen section analysis after the biopsy can help confirm the adequacy of the sample [2]. CT-guided biopsy has supplanted the need for an open biopsy, but a negative result should ideally be reconfirmed with repeat biopsy or an open procedure when the option is available, especially when evaluating sclerotic lesions $[1,2]$. The presence of a more accessible site for biopsy should be evaluated before proceeding for a spine biopsy.

\section{Blood investigations}

The hematologic work up is of significance in evaluating the nature of the lesion. Chronic infections like tuberculosis can closely mimic a metastatic lesion on the imaging. The hematologic profile can give a hint of the etiology, although getting a tissue diagnosis is gold standard. A relevant laboratory evaluation should include complete blood count, erythrocyte sedimentation rate, and C-reactive protein levels for evaluation of reactive and inflammatory processes, markers of specific disease, such as prostate-specific antigen and serum/urine protein electrophoresis can be considered in appropriate situations. Calcium, phosphorus, alkaline phosphatase levels, and basic metabolic panel are helpful in identifying certain disease processes and the related complications [5]. A variety of disease specific investigations are available, the detailed discussion of which is beyond the scope of this article.

\section{Treatment Goals in Spinal Metastasis}

The role of treatment in spinal metastasis is essentially palliative. Decisions are made not with the intent to prolong survival, but to improve quality of life in the final stages of the disease. Pain alleviation and preservation or recovery of neurological function is the two main goals of therapy in spinal metastasis. In addition, the choice of therapies is aimed at minimizing morbidity of treatment as well as achieving durable local tumor control.

The treatment of patients with metastatic spinal disease is complex and hence no single treatment protocol can be generalizable. The treatment decisions are highly individualized and are best taken by a multidisciplinary team of medical and radiation oncologists in collaboration with a spine surgeon with expertise and experience in dealing with spinal metastasis.

\section{Evolution of Treatment}

Before radiation became available, simple laminectomy was the only treatment for metastatic epidural spinal cord compression (MESCC). Introduction of radiotherapy saw improvement in outcome in approximately $50 \%$ patients, however only a few non-ambulatory patients ever walked again. Surgery went into disrepute when several studies showed laminectomy either alone or with radiotherapy did not improve outcomes 
predicted survival was $\leq 6$ months when score was $0-8, \geq 6$ months for score 9-11, and $\geq 1$ year for score $\geq 12$ [14].

Surgery is indicated in patients presenting with mechanical spinal instability irrespective of the radiosensitivity of the tumor or the degree of spinal cord compression (ESCC). Standalone radiotherapy is ineffective in treating symptoms related to spinal instability. The Spine Oncology Study Group (SOSG) has defined spinal instability as the "loss of spinal integrity as a result of a neoplastic process that is associated with movement-related pain, symptomatic or progressive deformity, and/or neural compromise under physiologic loads" [16]. They have also come up with a scoring system, the Spine instability neoplastic score (SINS) to help identify potentially unstable spinal metastases [16]. The scoring is based on the location, pain, bone lesion, collapse, spinal alignment, and posterolateral involvement with a score of 0-6 indicating stability without need for surgical stabilization and 13-18 indicating instability and need for surgical stabilization. Intermediate scores of 7-12 are indeterminate and need further evaluation for deciding appropriate treatment [16].

Table 1 shows common scoring systems used in vertebral metastatic disease for the assessment of the prognosis, survival, and the spinal stability.

Patchell et al. (2005) in a landmark prospective randomized trial assigned 50 patients with metastasis and spinal cord compression to surgery and radiotherapy and 51 patients to radiotherapy alone, both groups received ten 3 Gy fractions [10]. The primary outcome measure being ability to walk. The authors stopped the study midway because more patients in the surgery with radiotherapy group were able to walk after treatment $84 \%$ versus $57 \%$ in the radiotherapy group. They also retained ambulatory potential for a longer time (median 122 days vs. 13 days, $\mathrm{P}=0.003$ ). Of the 32 patients who were unable to walk, $62 \%$ in the surgery and radiotherapy group regained ambulatory potential versus $16 \%$ in the radiation group $(\mathrm{P}=0.01)$. The surgical approach depended on the location and level of compression. For anteriorly located tumors an anterior approach was used for laterally located tumors a lateral approach was used and for posteriorly located tumors, a posterior approach decompression was done. Spinal stabilization was done, based on instability, with cement augmentation and /or bone graft and screws and rods [10].

Therefore, surgery is the treatment of choice also in patients who present with high grade ESCC with or without myelopathy. Surgery is used to decompress the spinal cord and stabilize the spine using instrumentation. This provides the separation between the tumor and the spinal cord to provide safe and effective delivery of SRS postsurgery for local tumor control. With advances in technology, these surgeries can be done using minimal access and percutaneous fixation to minimize the morbidity of the procedure [12].

Radiotherapy, cEBRT or SRS, is required after surgery to achieve durable local tumor control $[12,17]$. The sequence and the time interval of radiotherapy are variable. Patients with a poor prognosis can be treated with a short course of radiotherapy while patients with good performance status, oligometastatic disease, and controlled primary disease can be treated with a longer course based on radiation oncology protocols.

NOMS framework and algorithm is based on neurology, oncology, mechanical and systemic assessment to make decisions regarding surgical, radiation therapy and oncological treatment [17]. The neurological assessment is based on the ESCC (low grade ESCC, high grade ESCC), myelopathy, and radiculopathy. The oncology assessment is based on the primary diagnosis (radiosensitive vs. radio-resistant) and response to various treatment options, that is EBRT, surgery, SRS, chemotherapy, or hormonal therapy. The mechanical assessment is for pathological fractures (based on stability, and instability) to decide on need for bracing or fixation. The systemic assessment (based on ability or inability to tolerate surgery) factors in the histopathology, grade of tumor, other systemic co-morbidities and expected survival, to help determine usefulness of the available treatment to the patient. Accordingly, decisions for EBRT, surgery, and SRS or a combination of two modalities can be made.

\section{Role of Cement Augmentation}

Vertebral augmentation with Vertebroplasty and Kyphoplasty, as a palliative method of treatment, has been shown to reduce pain and opioid analgesic requirements due to metastatic vertebral compression fractures [18]. A careful assessment of the mechanical spinal stability is important to address the need for a major spinal instrumented stabilization in these scenarios.

In essence, the management of spinal metastasis has evolved significantly over the past few decades. Advancement of protocols with the surgical treatment, radiation therapy and systemic therapy along with a systematic assessment of the various patient related factors forms the cornerstone of the management.

Figure 2 shows algorithm for the management of spinal metastasis involving a multidisciplinary approach.

\section{Conclusion}

A multidisciplinary systematic approach with consideration of oncology, radiology, neurology, and systemic comorbidities for expected survival should be used for planning appropriate treatment of spinal metastasis. The nature of the treatment should be individualized to the patient, the coexistent factors, and the available treatment resources. 
compared to radiotherapy alone. Radiation oncologists became reluctant to involve surgeons in the treatment decisions. Surgeons soon learnt that laminectomy was not the right surgical procedure. Laminectomy involved removal of posterior elements in a disease that involved predominantly the anterior column (vertebral body). The resulting spinal instability compromised the outcomes. In the $1980 \mathrm{~s}$, instrumented stabilization and reconstruction of anterior column was added to the decompression surgery. Surgery finally made a comeback when in 2005, Patchell et al., in a randomized controlled trial proved that surgical decompression and stabilization in combination with radiation was superior to radiation alone in spinal metastasis [10]. Last decade has seen tremendous technological innovations with the introduction of intensity-modulated radiotherapy image guided radiotherapy, stereotactic radiosurgery (SRS)/ gamma knife surgery $[11,12,13]$. These have the capability to deliver highly conformal radiation to spinal tumors and have modified the surgical indications established by the Patchell study. As this multimodal treatment armamentarium evolves further, the paradigms for treating spinal metastasis will continue to change.

\section{Role of Chemotherapy}

Chemotherapy has very limited role and is generally reserved for chemo-sensitive tumors with spinal metastases causing little or no symptoms. It is used mainly as an adjunct to other modalities in patients with symptomatic spinal metastases. Chemotherapy may be given for a recurrence after previous irradiated cord compression who is not a candidate for further radiotherapy or surgery [11].

\section{Role of Radiotherapy}

Radiotherapy is the least invasive modality for achieving durable local tumor control and is the mainstay of treating spinal metastasis. Conventional external beam radiotherapy (cEBRT) delivers fractionated radiation to the tumor without accurate conformal techniques. Due to this the adjacent spinal cord is also irradiated along with the tumor. The spinal cord is able to tolerate up to approximately $30 \mathrm{~Gy}$. cEBRT can achieve durable local control rates in histologies that respond favorably to a dose lower than this limit imposed by the spinal cord tolerance. Hematological malignancies (lymphoma, multiple myeloma, and plasmacytoma), seminoma and small cell cancers are extremely radiosensitive. Other solid tumors show varying degrees of radiosensitivity. Breast, ovarian, and prostate carcinomas are usually radiosensitive while renal, thyroid, hepatocellular, non-small cell, and colorectal cancers are radio-resistant to cEBRT $[12,13]$.

Patients with radiosensitive spinal metastasis presenting with biologic pain with minimal or no epidural compression (without neurological deficit) are ideal candidates for cEBRT. Malignancies that are extremely radiosensitive can be effectively treated with cEBRT alone even if presenting with high grade ESCC or myelopathy [12]. In patients in whom surgery is not an option due to limited life expectancy and medical co-morbidities, cEBRT can be an alternative. cEBRT is contraindicated in patients with previous radiation to prevent radiation induced spinal cord dysfunction. Any form of radiotherapy is generally ineffective in mechanical spinal pain secondary to instability or fracture. Maranzano et al. performed a randomized trial in patients with MESCC to assess two radiation fractionation schemes: A short course of two doses of 8 Gy with a 6-day break versus three doses over 2 weeks of 5 Gy each followed by five doses of 3 Gy with a break of 4 days between doses [13]. Treatment with the short course versus the split course of radiotherapy resulted in similar rates of back pain relief, maintenance of the ability to walk and good bladder function so the authors recommend that a schedule of two doses, each of $8 \mathrm{~Gy}$, should be used for patients with MESCC [13]. However, the trial compared only two non-standard shortcourse schedules and did not compare them with a longer standard schedule. With the advent of image guided stereotactic techniques, we now have the ability to target higher doses precisely on the tumor whilst sparing the spinal cord and other adjacent organs. Thus tumors that were traditional considered radioresistant can now be treated with SRS [12]. Even patients who have previously been treated with cEBRT are suitable candidates for SRS.

However, SRS use is limited in metastasis with high grade ESCC. In these situations, the tumor and the spinal cord are in such close proximity that the requisite dose cannot be delivered to the tumor without risking spinal cord toxicity. Therefore, SRS is ideal for patient with radio-resistant metastasis without significant epidural extension of the tumor [12].

\section{Role of Surgery}

Before surgery is considered it is important to evaluate if patient can tolerate surgery and enjoy the benefit of the surgery with at least 3-6 months survival. Unfortunately, physicians and surgeons may tend to over-estimate patient survival, so before deciding treatment modalities, patients' needs a thorough assessment.

The Tokuhashi score is one of the popularly used scores for making this assessment $[14,15]$. It has six components with three levels each, mainly general condition (based on Karnofsky performance status), spinal metastases, bone metastases, primary lesion, metastases to other organs, and neurological deficit. In the revised version, the primary lesion staging was changed from 3 to 6 levels, and the 
Declaration of patient consent: The authors certify that they have obtained all appropriate patient consent forms. In the form, the patient has given his consent for his images and other clinical information to be reported in the Journal. The patient understands that his name and initials will not be published, and due efforts will be made to conceal his identity, but anonymity cannot be guaranteed.

Conflict of Interest: NIL; Source of Support: NIL

\section{References}

1. Rose PS, Buchowski JM. Metastatic disease in the thoracic and lumbar spine: Evaluation and management. J Am Acad Orthop Surg 2011;19:37-48.

2. White AP, Kwon BK, Lindskog DM, Friedlaender GE, Grauer JN. Metastatic disease of the spine. J Am Acad Orthop Surg 2006;14:587-98.

3. Kirchhoff SB, Becker C, Duerr HR, Reiser M, Baur-Melnyk A. Detection of osseous metastases of the spine: Comparison of high resolution multi-detector-CT with MRI. Eur J Radiol 2009;69:567-73.

4. Edelstyn GA, Gillespie PJ, Grebbell FS. The radiological demonstration of osseous metastasis: Experimental observations. Clin Radiol 1967;18:158-62.

5. Tatsui H, Onomura T, Morishita S, Oketa M, InoueT. Survival rates of patients with metastatic spinal cancer after scintigraphic detection of abnormal radioactive accumulation. Spine (Phila Pa 1976) 1996;21:2143-8.

6. Rougraff BT, Kneisl JS, Simon MA. Skeletal metastases of unknown origin: A prospective study of a diagnostic strategy. J Bone Joint Surg Am 1993;75:1276-81.

7. Bredella MA, Essary B, Torriani M, Ouellette HA, Palmer WE. Use of FDG-PET in differentiating benign from malignant compression fractures. Skeletal Radiol 2008;37:405-13.

8. Metser U, Lerman H, Blank A, Lievshitz G, Bokstein F, EvenSapir E. Malignant involvement of the spine: Assessment by 18F-FDG PET/CT. J Nucl Med 2004;45:279-84.

9. Lis E, Bilsky MH, Pisinski L, Boland P, Healey JH, O'malley B, et al. Percutaneous CT-guided biopsy of osseous lesion of the spine in patients with known or suspected malignancy. AJNR Am J Neuroradiol 2004;25:1583-8.

10. Patchell RA, Tibbs PA, Regine WF, Payne R, Saris S, Kryscio
$\mathrm{RJ}$, et al. Direct decompressive surgical resection in the treatment of spinal cord compression caused by metastatic cancer: A randomised trial. Lancet 2005;366:643-8.

11. Cole JS, Patchell RA. Metastatic epidural spinal cord compression. Lancet Neurol 2008;7:459-66.

12. Sharan AD, Szulc A, Krystal J, Yassari R, Laufer I, Bilsky MH. The integration of radiosurgery for the treatment of patients with metastatic spine diseases. J Am Acad Orthop Surg 2014;22:447-54.

13. Maranzano E, Bellavita R, Rossi R, de Angelis V, Frattegiani A, Bagnoli R, et al. Short-course versus split-course radiotherapy in metastatic spinal cord compression: Results of a Phase III, randomized, multicenter trial. J Clin Oncol 2005;23:3358-65.

14. Tokuhashi Y, Matsuzaki H, Oda H, Oshima M, Ryu J. A revised scoring system for preoperative evaluation of metastatic spine tumor prognosis. Spine (Phila Pa 1976) 2005;30:2186-91.

15. Tokuhashi $Y$, Uei $H$, Oshima M, Ajiro $Y$. Scoring system for prediction of metastatic spine tumor prognosis. World J Orthop 2014;5:262-71.

16. Fisher CG, DiPaola CP, Ryken TC, Bilsky MH, Shaffrey $\mathrm{Cl}$, Berven $\mathrm{SH}$, et al. A novel classification system for spinal instability in neo-plastic disease: An evidence-based approach and expert consensus from the spine oncology study group. Spine (Phila Pa 1976) 2010;35:E1221-9.

17. Laufer I, Rubin DG, Lis E, Cox BW, Stubblefield MD, Yamada Y, et al. The NOMS framework: Approach to the treatment of spinal metastatic tumors. Oncologist 2013;18:744-51.

18. Health Quality Ontario. Vertebral augmentation involving vertebroplasty or kyphoplasty for cancer-related vertebral compression fractures: A systematic review. Ont Health Technol Assess Ser 2016;16:1-202.
Conflict of Interest: NIL

Source of Support: NIL

\section{How to Cite this Article}

Badve S, Dhawale A, Chaudhary K, Anchan C. A multidisciplinary approach to the management of spinal metastasis: A review article. Journal of Clinical Orthopaedics July-Dec 2021;6(2):16-22. 